\title{
Single Incision Laparoscopy, performance and current status in surgical practice
}

\section{Dear Editor,}

The technique of single-incision laparoscopic surgery(SILS) is developed based on that of conventional laparoendoscopic surgery. At first, surgeons wanted to devise a method for visceral surgery with no incision on body's surface, which is called natural orifice transluminal endoscopic surgery (NOTES). As early as the 1970s, Dr. Raimund Wittmoser, the "father" of modern thoracoscopic surgery of the autonomic nervous system, used a singleintercostal incision through which he inserted a multifunctional port which contained all the instruments and optics. Another example of SILS in early times is the application of laparoscopic instrumentation to resect lesions in the rectum or sigmoid colon. Using the anus as the portal of entry, trans anal endoscopic microsurgery (TEMS) employs a specialized multichannel trocar to reach lesions located 8 to $18 \mathrm{~cm}$ away from the anal verge. Compared to NOTES, SILS is easier, safer, more applicable and convenient for surgeons to master as laparoscope has been a tool for surgeons for a long time.

\section{Advantages of SILS}

The typical characteristic of SILS operation is the use of a single trocar site where multiple laparoscopic instruments are manipulated via a multiport system. Ports typically contain three or four channels. The later often affords the ability to place a dedicated retractor. Placement of the trocar at the umbilicus allows for minimal visible scar and thus more aesthetically appealing than traditional laparoscopic surgical scars. Besides, incision in the umbilicus, a preexisting scar, is thought to be less painful, have fewer wound complications, and leads to quicker return to activity than conventional laparoscopy.

\begin{tabular}{l|l} 
Access this article online \\
Website:
\end{tabular}

\section{Disadvantages of SILS}

There are many challenges faced by the operating surgeon in SILS procedures. These include crowded trocar placement, a lack of triangulation of left and right-hand instruments, frequent crossing or clashing of instruments, limited visualization, and limited retraction ability. The two operation ports are close to the observation port (the distance between them can be less than $10 \mathrm{~mm}$ ), and they often form a triangle (an inverted triangle in upper abdominal operation and an equilateral one in lower abdominal operation). These three ports interfere with each other, influencing exposure of the operation fields and effective tissue separation, which is called "Chopsticks effect". These challenges are mitigated by surgeon experience and the development of specialized instruments. Articulating or curved instruments of varying lengths and an extended length can improve working space. Curved instruments are typically reusable and offer less clutter than their more sophisticated counterparts, providing some cost reduction. A low-profile HD scope with or without a deflectable tip can improve visualization greatly. Even with such instrumentation, the learning curve is very steep, particularly when the surgeon is forced to work in a crosshanded technique ${ }^{[1]}$

Ideal patients for SILS:The ideal patient for SILS is one with lower body mass index (BMI), early disease, and no previous abdominal surgery. Contraindications include those true of traditional laparoscopy. Relative

\section{Correspondence:}

Dr Javid Ahmad Bhat,

Fellow Minimal Access surgery.

Department of General and Minimal invasive surgery, SKIMS, Soura.

Email:drjavidbhat@gmail.com

How to cite this article: Bhat JA, Malik AA, Wani MA, Mir IN. Single Incision Laparoscopy Performance and Current Status in Surgical Practice. jms 2019;22(2):46-48

Received: 07-12-2019 Accepted: 23-12-2019 
Bhat JA; et al; Single Incision Laparoscopy, performance and current status in surgical practice

contraindications include previous surgery and high body mass index (BMI). Patients with a high BMI or central obesity can pose a challenge because the umbilicus may be located far from operative target. Size and morphology of the target organ should always be considered when doing SILS. As safety should always be the paramount concern, the addition of extra trocars or conversion to traditional laparoscopy should not be considered a failure

\section{Performance of SILS in comparison to Conventional laparoscopy}

As laparoscopic skills improve and technologies advance, SILS was suggested as an alternative technology totraditional laparoscopy for reducing post ${ }^{-}$operative pain and improving cosmetic results and was applied in a variety of general surgery cases. Many studies have evaluated the feasibility and safety of this type of surgery including reviews and meta- analysis ${ }^{2}$. However, it is questionable what the full benefit of the dramatic reduction in ergonomics and the increase in complexity provide beyond an improved cosmetic appearance. This is in large part due to the already improved benefits of laparoscopic surgery. For these reasons, although many abdominal surgeries can be performed using SILS, many surgeons are skeptical on its practicality for routine use over traditional laparoscopic surgery.

\section{SILS appendectomy}

SILS appendectomy has been described in many pediatric cases, but as the patient size and weight increase, so does the difficulty of the procedure and conversion rates. Metaanalysis of randomized control studies in adults have showed longer operating time and higher rate of conversion. There were no differences between the two groups in visual analogue pain scores, doses of analgesics, overall complication rates, wound infection, or cosmesis. In general, SILS- Appdendectomy is more technologically difficult and results in a longer operative time, with no clear consensus on this trend ${ }^{3,4}$ Surgery of spleen, liver and stomach may cost more time by the former one. It requires surgeons to accumulate more experience on these surgeries. All in all, the SILS results in a better cosmetic outcome and more minimally invasive

\section{SILS cholecystectomy}

Comparable results have been reported for SILS versus traditional four-port cholecystectomy. It has gained attention as an alternative minimally invasive surgery with a shorter learning curve Moreever, due to its minimally invasive nature, it may be more acceptable and with higher satisfaction because of single wound $\mathrm{d}^{5}$ Acute cholecystitis is a factor associated with a lower success rate 59.9 vs $93.0 \%$ and longer operative time of 78 vs $70 \mathrm{~min}$. A BMI $>30$ was also associated with longer operative times ${ }^{6 .} A$ recent metaanalysis pooling ten randomized control studies evaluated SILC vs laparoscopic cholecystectomy and found that although there were improved postoperative pain and cosmesis scores, there was a significant increase in major complications (CBD injury, requirement for re-exploration, and large vessel injury) with a relative risk of 3.0 as well as an increase in minor complications. Operation times were significantly longer in the SILC group with a mean difference added time of $23 \mathrm{~min}$. No difference was noted in requirement for conversion to open or addition of extra portsites were noted ${ }^{7}$ A meta-analysis performed by Ahmed and colleagues in 2010 found the conversion rate from SILS to conventional laparoscopy to be $0 \%$ to $24 \%$ for cholecystectomies, $0 \%$ to $41 \%$ for appendectomies, and $0 \%$ to $33 \%$ for nephrectomies. The most common complications were intra-abdominal abscesses and wound infections.

\section{SILS colorectal}

Conventional laparoscopic surgery (CLS) have shown that short-term clinical and oncological outcomes of SILS are better than that of CLS ${ }^{8}$ Liet al. had very fully confirmed that SILS had less blood loss, shorter incision length and hospital staybut longer operative time for colorectal disease ${ }^{9}$ With respect to the conversion rate to open surgery, SILS is similar with CLS. The main reasons which could impact conversion rate including obesity, narrow pelvis, important vascular variation, vascular injury, and hypertrophic mesentery ${ }^{10}$ But for sigmoid colon cancer, SILS had a shorter operation time and hospital stay than CLS due to good location of sigmoid colon cancer. Few studies have evaluated readmission, local recurrence and distant metastasis which showed SILS and CLS had similar results ${ }^{11}$ Subgroup analysis of region showed that SILS had better outcomes than CLS, including complication rate, incision length, defecation time, exhaust time, and hospital stay for eastern patients, and SILS had more lymph node resection for western patients than CLS. Western patients 
Bhat JA; et al; Single Incision Laparoscopy, performance and current status in surgical practice

had a particularly difficult surgery with high body mass and narrow operation space.

\section{Current status of SILS}

Currently most of the abdominal surgeries which are performed through conventional laparoscopy can also be done by SILS with varying success and complication rates. the most common procedures are single- port laparoscopic cholecystectomies, colectomies andappendectomies ${ }^{12}$

Procedures in which SILS has overall good performance include appendectomy, cholecystectomy, colorectal cancers, splenectomy (non-portal hypertension and nongiant spleen),partial gastrectomy, liver cyst fenestration and cholecysto-jejunostomy. Procedures in which the performance is comparable or less satisfactory and which need further validation include hepatectomy, thyroidectomy, total gastrectomy, common bile duct exploration, distal pancreatectomy for benign lesions and cholangio-jejunostomy. Abdominal operations in which performance has been poor and is not recommended for SILS is pancreaticoduodenectomy and distal pancreatectomy for malignant lesions and semi hepatectomy. We expect this technique would be a powerful tool for surgeons in the near future and the robotics application may further provide the bridge necessary to bypass the significant technical skills learning curve required to operate through a single site

\section{REFERENCES}

1. Ahmed I, Cianco F, Ferrar V, et al. Current status of single incision laparoscopic surgery: European experts' views. SurgLaparoscEndoscPercutan Tech. 2012;22(3):194-199

2. Lv $\mathrm{C}, \mathrm{Wu} \mathrm{S}, \mathrm{Wu} \mathrm{Y}$, Shi J, Su Y, Fan $\mathrm{Y}$, et al. Single-incision laparoscopic versus traditional multiport laparoscopic colorectal surgery A cumulative meta-analysis and systematic review. Int $\mathbf{J}$ Colorectal Dis 2013;28:611-21.

3. Fan $\mathrm{Y}, \mathrm{Wu}$ SD, Kong J, Chao W. Single-incision laparoscopic splenectomy with conventional instruments: Preliminary experience in consecutive patients and comparison to standard multiple-incision laparoscopic splenectomy. J LaparoendoscAdvSurg Tech A 2014;24:799-803.

4. Shao XJ. Comparative study of single-port laparoscopic splenectomy with conventional laparoscopic splenectomy surgery. China $\mathrm{J}$ Endosc 2014;20:805-7.

5. Teubner $\mathrm{O}$ et al .prospective study comparing quality of life and cosmetic results between single port and conventional laproscopic cholecystectomy, Surgsci 2016,7,114 to 25 .

6. Antoniou SA, Pointner R, Granderath FA. Singleincision laparoscopic cholecystectomy: a systematic review. SurgEndosc. 2011;25(1):36777.

7. Evers L, Bouvy N, Branje D, Peeters A. Singleincision laparoscopic cholecystectomy versus conventional four-port laparoscopic cholecystectomy: a systematic review and meta-analysis. SurgEndosc. 2016;31(9):343748.

8. Brockhaus AC, Sauerland S, Saad S. Single-incision versus standard multiincision laparoscopic colectomy in patients with malignant or benign colonic disease: a systematic review, meta-analysis and assessment of evidence. BMC Surg. 2016;16:71.

9. Li HJ, Huang L, Li TJ, et al. Short-term outcomes of single-incision versus conventional laparoscopic surgery for colorectal diseases: meta-analysis of randomized and prospective evidence. J Gastrointest Surg. 2017;21:193145.

10. Yu H, Shin JY. Short-term outcomes following reduced-port, single-port, and multi-port laparoscopic surgery for colon cancer: tailored laparoscopic approaches based on tumor size and nodal status. Int J Color Dis. 2016;31:11522.

11. Hirano Y, Hattori M, Douden K, et al. Single-incision laparoscopic surgery for colorectal cancer. World J Gastrointest Surg. 2016;8:95100.

12. Keller DS, Ibarra S, Flores GJ, et al. Outcomes for single-incision laparoscopic colectomy surgery in obese patients: a case-matched study. SurgEndosc. 2016;30:73944.

'Javid Ahmad Bhat, ${ }^{2}$ Ajaz Ahmad Malik, ${ }^{3}$ Munir Ahmad Wani, ${ }^{4}$ Irfan Nazir Mir

1,4Fellow Minimal Access Surgery,

${ }^{2,3}$ Professor, Additional Professor

Department of General and Minimal Invasive Surgery, SKIMS Soura 